\title{
Percepción entre médicos bogotanos del riesgo de reacciones adversas a grupos de medicamentos de uso común
}

\author{
Jefferson Antonio Buendía, Andrés F. Zuluaga \\ Departamento de Farmacología y Toxicología, Facultad de Medicina, Universidad de Antioquia, Medellín, Colombia
}

Introducción. Las reacciones adversas a medicamentos son un problema de salud pública; sin embargo, todavía son muchos los médicos que no parecen reconocer el riesgo asociado al uso de medicamentos.

Objetivo. Valorar la percepción del riesgo de reacciones adversas asociado a los grupos de medicamentos más frecuentemente utilizados por los profesionales de la medicina en la práctica clínica.

Materiales y métodos. Se llevó a cabo un estudio descriptivo y transversal con 200 médicos con un mínimo de dos años de experiencia clínica, y vinculados a hospitales públicos o clínicas privadas de Bogotá. La percepción del riesgo asociado a las reacciones adversas a medicamentos de 31 grupos farmacológicos o terapéuticos, se valoró usando una escala analógica visual (0-10 puntos).

Resultados. La percepción del riesgo fue baja (puntaje promedio <5) con relación a 19 (61\%) de los 31 grupos de medicamentos. Los tres grupos que se relacionaron con una mayor percepción de riesgo de reacciones adversas a medicamentos (puntaje promedio $>7$ ) fueron: (a) la quimioterapia para el cáncer (mediana=8,5; rango intercuartílico: 1,8), (b) la insulina (mediana=8; rango intercuartílico: 4,8) y (c) los anticoagulantes (mediana=7,5; rango intercuartílico: 4).

Conclusiones. Aunque existe una relación adecuada entre la percepción del riesgo y la frecuencia de las reacciones adversas a los medicamentos asociada con los grupos de mayor puntaje, también existe una importante subestimación del riesgo en medicamentos de uso común a nivel hospitalario y ambulatorio, como es el caso de los antiinflamatorios no esteroideos, los antihipertensivos y los anticonceptivos orales.

Palabras clave: preparaciones farmacéuticas, toxicidad, efectos colaterales y reacciones adversas relacionados con medicamentos, control de riesgos, utilización de medicamentos.

http://dx.doi.org/10.7705/biomedica.v34i3.2181

Physicians' insight about adverse drug reaction to frequently used medication groups in Bogotá (Colombia)

Introduction: Adverse drug reactions are a public health problem; however, still many prescribers do not seem to recognize the risk associated with the use of medications.

Objective: To assess the perception of the risk of adverse reactions associated with the groups of drugs most frequently used in clinical practice.

Materials and methods: Descriptive study made in 200 physicians with at least 2 years of clinical experience in Bogota, Colombia. The risk of adverse drug reactions associated with the use of medications was assessed using a visual analog scale (0-10 points).

Results: The perception of risk was $<5$ points for 19 of the $31(61 \%)$ therapeutic groups. The therapeutic groups that were related to increased perception of risk were chemotherapy for cancer (median, 8.5, interquartile range: 1.8), insulin (median, 8, interquartile range: 4.8) and anticoagulants (median, 7.5, interquartile range: 4 ).

Conclusions: Although there is a relationship between the perception of risk and the frequency of adverse drug reactions associated with therapeutic groups of higher score, there is also a strong underestimation of the risk of medicines in common use at hospital and ambulatory level such as NSAID, antihypertensive drugs and oral contraceptives.

Key words: Pharmaceutical preparations, toxicity, drug-related side effects and adverse reactions, risk management, drug utilization.

http://dx.doi.org/10.7705/biomedica.v34i3.2181

Contribución de los autores:

Ambos autores contribuyeron de igual manera al diseño, análisis y elaboración del manuscrito. 
La expresión latina primum non nocere (sobre todo, no causar daño) resume un principio general de la medicina (1). Cuando una persona usa un medicamento puede obtener un resultado favorable (benéfico) o deletéreo (nocivo). Aunque la mayoría de los médicos y los pacientes buscan alcanzar el efecto benéfico de los medicamentos, es más probable que las intervenciones en que se emplean generen uno o más efectos nocivos (2-4).

En este sentido, desde hace más de 40 años la Organización Mundial de la Salud viene llamando la atención sobre la importancia de investigar y educar en este campo (5); sin embargo, los profesionales de la salud en todo el mundo aún siguen haciendo millones de prescripciones sin considerar como un problema relevante las reacciones inesperadas y perjudiciales a los medicamentos (o reacciones adversas a los medicamentos) (6), ocasionando, así, enormes pérdidas humanas y económicas. Por ejemplo, Ernst y Grizzle estimaron que en el 2000, el costo de la morbilidad y la mortalidad relacionadas con medicamentos sobrepasó los USD\$ 177 mil millones en los Estados Unidos (4). Actualmente, no hay duda de que las reacciones adversas a medicamentos constituyen un problema de salud pública mundial, aunque existe un gran subregistro en los países en desarrollo (7-11). En un estudio llevado a cabo en Colombia, se encontró que alrededor del $43 \%$ de los profesionales de la salud no reportaba sistemáticamente los eventos adversos, incluso $21 \%$ de los entrevistados reconoció no haber realizado nunca este tipo de reportes (12).

La habilidad para percibir condiciones externas peligrosas y la capacidad de responder a ellas, constituyen un elemento esencial para la supervivencia de cualquier especie (13). Los estudios de percepción del riesgo se han diseñado con el fin de examinar la opinión que expresan las personas en su respuesta a preguntas orientadas a caracterizar y evaluar actividades o tecnologías peligrosas (14). La percepción del riesgo asociado al uso de medicamentos, específicamente entre el personal de salud, es fundamental para aumentar la notificación de eventos, tomar mejores decisiones y lograr un impacto positivo en los costos

Correspondencia:

Jefferson Antonio Buendía, Departamento de Farmacología y Toxicología, Facultad de Medicina, Universidad de Antioquia, Carrera 51D N 62-29, Medellín, Colombia

Teléfono: (574) 2196020

jefferson.buendia@gmail.com

Recibido: 23/10/13; aceptado: 03/05/14 relacionados con las reacciones adversas a medicamentos $(15,16)$. Hasta el momento se han desarrollado algunos estudios sobre la percepción de este riesgo entre profesionales de la salud en Europa, pero, hasta donde sabemos, no se ha llevado a cabo ningún estudio similar en nuestro país (17-20).

El objetivo del presente trabajo fue valorar, mediante una escala analógica visual, la percepción que los profesionales de la salud tienen del riesgo de reacciones adversas asociado a los grupos de medicamentos más frecuentemente utilizados en la práctica clínica.

\section{Materiales y métodos \\ Diseño y población}

Se adelantó un estudio descriptivo y transversal entre 200 médicos que tenían, al menos, dos años de experiencia clínica y laboraban en hospitales públicos o clínicas privadas de la ciudad de Bogotá. El reclutamiento de los participantes se hizo mediante invitación escrita enviada por el comité de vigilancia farmacológica de cada una de las instituciones participantes: cuatro hospitales públicos y cuatro clínicas privadas. Todos los participantes recibieron una explicación completa del objetivo del estudio antes de ingresar en él y sobre el carácter anónimo de sus respuestas. A quienes no respondieron a la primera invitación después de 30 días de haberla remitido, se les envió una segunda invitación a participar en el estudio.

\section{Mediciones}

La percepción del riesgo asociado a las reacciones adversas a 31 grupos de medicamentos de uso común, se valoró usando la escala analógica visual publicada por Bongard, et al. (20). Se evaluó la percepción del riesgo de cada grupo de medicamentos de uso común midiendo la distancia entre el extremo izquierdo de la escala (igual a cero) y la marca hecha por el participante. Dado que la escala tiene $10 \mathrm{~cm}$, el riesgo percibido se midió con un puntaje cuantitativo de 0 (mínimo riesgo percibido) a 10 (máximo riesgo percibido). El estudio fue aprobado por el comité de ética de uno de los hospitales participantes.

\section{Análisis estadístico}

En el caso de las mediciones cuantitativas, los resultados se expresaron en medianas y rango intercuartílico, y, en el caso de las mediciones cualitativas, como proporciones. El análisis estadístico se hizo con el software Stata 11.0. Con un 
Cuadro 1. Características sociodemográficas y educativas de los médicos encuestados

\begin{tabular}{lrc}
\hline Variable & $\mathbf{n}$ & Media (\%) \\
\hline Edad (media) & & 36,84 \\
Sexo & & \\
$\quad$ Femenino & 82 & 41,00 \\
$\quad$ Masculino & 118 & 59,00 \\
Año de graduación & & \\
$\quad 1977-1990$ & 85 & 42,50 \\
$1990-2000$ & 65 & 32,50 \\
$2000-2008$ & 50 & 25,00 \\
Experiencia administrativa & 128 & 64,10 \\
Experiencia docente & 67 & 33,30 \\
Formación & & \\
$\quad$ Sin posgrado & 67 & 33,30 \\
$\quad$ Especialidad médico-quirúrgica & 56 & 28,21 \\
$\quad$ Especialidad administrativa o relacionada & 77 & 38,49 \\
$\quad$ con la salud pública & \multicolumn{2}{c}{} \\
\hline
\end{tabular}

tamaño muestral de 200 participantes, el intervalo de confianza fue de $95 \%$, la precisión de $6 \%$, y la desviación estándar de $\pm 0,5$ en los puntajes promedio obtenidos.

\section{Resultados}

Se enviaron 350 invitaciones; solo el $58 \%$ de los invitados respondieron y participaron en el estudio, cifra esta similar a la de estudios previos al nuestro (46 a $51 \%)(20)$.

En el cuadro 1 se aprecia la caracterización básica de los 200 médicos participantes. El promedio de edad fue de 37 años, la mayoría eran hombres (59\%), graduados de pregrado después de 1990 (58\%), con experiencia administrativa (64\%) y con algún posgrado (67\%).

En el cuadro 2 se resume la valoración dada por los encuestados de la percepción de riesgo de reacciones adversas a grupos de medicamentos de uso frecuente. La percepción del riesgo fue baja (puntaje promedio <5) para 19 de los $31(61 \%)$ grupos de medicamentos, y el puntaje más bajo se observó con los antiparasitarios (mediana $=4$, rango intercuartílico: 4). Los tres grupos de medicamentos que se relacionaron con una mayor percepción de riesgo de reacciones adversas (puntaje promedio >7) fueron: (a) la quimioterapia para el cáncer (mediana=8,5, rango intercuartílico: 1,8), (b) la insulina (mediana $=8$, rango intercuartílico: 4,8) y (c) los anticoagulantes (mediana $=7,5$; rango intercuartílico: 4).

\section{Discusión}

La modificación en la percepción del riesgo constituye uno de los pilares de la gestión del riesgo en salud. Dada la problemática que plantea el uso indiscriminado de medicamentos y el aumento de la morbilidad causada por las reacciones adversas a ellos, lograr que los profesionales de la salud relacionen la percepción del riesgo con la frecuencia de dichas reacciones reportada en los estudios clínicos constituye, sin lugar a dudas, un reto para los sistemas de vigilancia farmacológica y las entidades académicas $(21,22)$.

Aunque en nuestro estudio hubo concordancia entre la percepción del riesgo de algunos grupos de medicamentos, como los quimioterapéuticos, la insulina, los anticoagulantes y los trombolíticos, y los reportes actuales de reacciones adversas a medicamentos en el país $(23,24)$, otros grupos importantes asociados con una alta morbilidad secundaria, como los antiinflamatorios no esteroideos (25), sorprendentemente se ubicaron por debajo de otros grupos con menor reporte de reacciones adversas, como los ansiolíticos o los antibióticos.

También, se observó una subestimación del riesgo de los medicamentos antihipertensivos y de los anticonceptivos orales, cuya percepción fue baja (puntaje promedio <5) comparada con lo reportado por estudios previos de vigilancia farmacológica en el país (24). Estos hallazgos concuerdan con los resultados de estudios previos, según los cuales los médicos perciben mayor riesgo en el uso de medicamentos para el sistema nervioso central, mientras que aquellos de uso frecuente, como los antiinflamatorios no esteroideos, los diuréticos y los anticonceptivos orales, se perciben con un menor potencial de causar reacciones adversas $(20,26)$.

Los factores que pueden explicar esta percepción equivocada del riesgo de reacciones adversas a estos medicamentos entre los profesionales de la salud y entre los pacientes, se han explorado solo parcialmente. Por ejemplo, Ganther, et al., estimaron que alrededor del $50 \%$ de los pacientes considera que el mayor riesgo de reacciones adversas de los medicamentos genéricos depende de la condición médica que deba ser tratada (27). Sin embargo, también se ha demostrado que la cantidad de información respecto a la seguridad del medicamento y su difusión a través de los medios de comunicación masiva, tienen un impacto en la percepción del riesgo de reacciones adversas entre los profesionales de la salud y los pacientes (28).

Lo anterior refuerza la importancia de fortalecer los programas de pregrado y de posgrado en farmacología clínica, de implementar cursos obligatorios 
Cuadro 2. Valoración de la percepción del riesgo de reacciones adversas a grupos de medicamentos de uso frecuente

\begin{tabular}{|c|c|c|c|c|}
\hline \multirow[t]{2}{*}{ Grupo de medicamentos } & \multirow[t]{2}{*}{ Mediana } & \multicolumn{2}{|c|}{ Rango } & \multirow{2}{*}{$\begin{array}{c}\text { Rango } \\
\text { intercuartílico }\end{array}$} \\
\hline & & máximo & mínimo & \\
\hline Quimioterapia para el cáncer & 8,5 & 10 & 4 & 1,8 \\
\hline Insulina & 8 & 10 & 0 & 4,9 \\
\hline Anticoagulantes & 7,5 & 10 & 1 & 4 \\
\hline Opioides & 7 & 10 & 3 & 1,5 \\
\hline Trombolíticos & 7 & 10 & 3 & 2 \\
\hline Hipnóticos & 7 & 9 & 2 & 3 \\
\hline Antidepresivos & 6,1 & 9 & 1 & 3 \\
\hline Antiarrítmicos & 6 & 9 & 3 & 2 \\
\hline Antipsicóticos & 6 & 9 & 4 & 3 \\
\hline Aspirina & 6 & 9 & 1 & 3,2 \\
\hline Antiepilépticos & 6 & 9 & 1 & 3,5 \\
\hline Ansiolíticos & 6 & 9 & 2 & 3,6 \\
\hline Antibióticos & 5,5 & 9 & 2 & 1,9 \\
\hline Hipoglucemiantes orales & 5,5 & 9 & 0 & 2,8 \\
\hline Antiasmáticos & 5,5 & 9 & 1 & 3,5 \\
\hline Antihistamínicos & 5,1 & 9 & 1 & 2 \\
\hline Antianginosos & 5 & 9 & 1 & 2 \\
\hline Antieméticos & 5 & 9 & 1 & 2 \\
\hline Antitusivos & 5 & 9 & 1 & 2,5 \\
\hline Antiinflamatorios no esteroideos & 5 & 9 & 1 & 3 \\
\hline Antihipertensivos & 5 & 9 & 0,5 & 3,5 \\
\hline Antiparkinsonianos & 5 & 9 & 1 & 3,5 \\
\hline Antivirales & 4,6 & 9,5 & 1 & 3 \\
\hline Antitiroideos & 4,6 & 10 & 1 & 3 \\
\hline Antifúngicos & 4,6 & 9 & 1 & 4 \\
\hline Terapia de reemplazo hormonal & 4,4 & 9 & 1 & 3,5 \\
\hline Antiulcerosos & 4 & 9 & 1 & 2 \\
\hline Hipolipemiantes & 4 & 9 & 1 & 2 \\
\hline Antianémicos & 4 & 9 & 1,4 & 2,5 \\
\hline Anticonceptivos orales & 4 & 9 & 1 & 3,5 \\
\hline Antiparasitarios & 4 & 9 & 1 & 4 \\
\hline
\end{tabular}

de análisis crítico de la literatura científica para profesionales de la salud, así como de regular la calidad y la cantidad de información a la que están expuestos los pacientes por parte de los productores y los distribuidores de medicamentos, con el fin de impedir la manipulación equivocada de dicha información $(29,30)$. Igualmente, nuestros resultados ponen de manifiesto la falta de estímulo para el reporte de estos eventos y la ausencia de retroalimentación entre las entidades reguladoras del sistema de salud y los profesionales en torno a los hallazgos de la vigilancia farmacológica, lo cual constituye una barrera, no solo para la prevención de las reacciones adversas a medicamentos, sino también para intensificar la cautela con respecto a aquellos con poco margen de seguridad.

Nuestro estudio tuvo algunas limitaciones. La tasa de respuesta a la convocatoria de médicos en cada hospital fue intermedia, similar a la encontrada en estudios previos (20). Aunque no es posible determinar cómo afectó este hecho los resultados obtenidos, es probable que los médicos asistentes estuvieran más sensibilizados frente al tema, lo que pudo llevar a sobrevalorar la percepción de riesgo.

Dada la naturaleza descriptiva y exploratoria del estudio, nuestro objetivo no fue determinar las causas de la subestimación o sobrestimación del riesgo de reacciones adversas a medicamentos, pero dicho aspecto amerita ser explorado en próximos estudios.

Por último, no sabemos si la herramienta de medición utilizada, una escala analógica visual comúnmente empleada en este tipo de estudios $(3,5)$, ha sido sometida a validación interna o externa, y tampoco pudimos identificar un instrumento específico que permitiera cuantificar directamente la percepción del riesgo de reacciones adversas, por lo que, a pesar de reconocer dicha limitación, consideramos que la escala era adecuada para desarrollar el estudio. 
En conclusión, los antineoplásicos, la insulina y los anticoagulantes son los grupos de medicamentos con mayor percepción de riesgo de reacciones adversas entre la población estudiada. Sin embargo, a nivel hospitalario y ambulatorio existe una importante subestimación del riesgo de reacciones adversas a medicamentos de uso común como los antiinflamatorios no esteroideos, los antihipertensivos y los anticonceptivos orales.

\section{Agradecimientos}

A la Universidad de Antioquia, Estrategia de Sostenibilidad, 2013-2014.

\section{Conflicto de intereses}

Los autores declaran no tener ningún conflicto de intereses.

\section{Financiación}

El trabajo se financió con fondos propios de los autores.

\section{Referencias}

1. van Haaren F. 'Primum non nocere': A review of Taking America off Drugs: Why behavioral therapy is more effective for treating ADHD, OCD, depression and other psychological problems by Stephen Ray Flora. Behav Anal Pract. 2009;2:58-62.

2. Kunac DL, Kennedy J, Austin N, Reith D. Incidence, preventability, and impact of Adverse Drug Events (ADE) and potential ADEs in hospitalized children in New Zealand: A prospective observational cohort study. Paediatr Drugs. 2009;11:153-60. http://dx.doi.org/10.2165/00148581 200911020-00005

3. Bates DW, Cullen DJ, Laird N, Petersen LA, Small SD, Servi D, et al. Incidence of adverse drug events and potential adverse drug events. Implications for prevention. ADE Prevention Study Group. JAMA. 1995;274:29-34. http://dx.doi.org/10.1001/jama.1995.03530010043033

4. Ernst FR, Grizzle AJ. Drug-related morbidity and mortality: Updating the cost-of-illness model. J Am Pharm Assoc (Wash). 2001;41:192-9.

5. Helling $\mathbf{M}$, Venulet $\mathbf{J}$. Drug recording and classification by the WHO research centre for international monitoring of adverse reactions to drugs. Methods Inf Med. 1974;13: 169-78.

6. Cosentino M, Leoni O, Oria C, Michielotto D, Massimo E, Lecchini S, et al. Hospital-based survey of doctors' attitudes to adverse drug reactions and perception of drug-related risk for adverse reaction occurrence. Pharmacoepidemiol Drug Saf. 1999;8(Suppl.1):S27-35.

7. Atuah KN, Hughes D, Pirmohamed M. Clinical pharmacology: Special safety considerations in drug development and pharmacovigilance. Drug Saf. 2004;27: 535-54.

8. Buendía-Rodríguez JA, López-Gutiérrez JJ, García-Vega OA, Díaz-Rojas J, Sánchez-Villamil JP. Drug prescription patterns in patients attended at a middle-and high-complexity level institution. Rev Salud Pública (Bogotá). 2008;10:60514. http://dx.doi.org/10.1590/S0124-00642008000400010

9. Tantikul C, Dhana N, Jongjarearnprasert K, Visitsunthorn $\mathbf{N}$, Vichyanond $\mathbf{P}$, Jirapongsananuruk $\mathbf{O}$. The utility of the World Health Organization-The Uppsala Monitoring Centre (WHO-UMC) system for the assessment of adverse drug reactions in hospitalized children. Asian Pac J Allergy Immunol. 2008;26:77-82.

10. Olsson S. International reporting of adverse drug reactions: The WHO program. Semin Dermatol. 1989;8:72-4.

11. Álvarez-Requejo A, Carvajal A, Bégaud B, Moride Y, Vega T, Arias LH. Under-reporting of adverse drug reactions. Estimate based on a spontaneous reporting scheme and a sentinel system. Eur J Clin Pharmacol. 1998;54:483-8.

12. Segura O, Pacific H. Frames of mind, practices and needs of the health professionals with regards to pharmacovigilance and pharmacoepidemiology: A descriptive study. Acta Med Colomb. 2003;28:181-4.

13. Slovic P. Perception of risk. Science. 1987;236:280-5. http://dx.doi.org/10.1126/science.3563507

14. Schlich T, Tröhler U. The risks of medical innovation: Risk perception and assessment in historical context. Routledge studies in the social history of medicine. Abingdon and New York: Routledge; 2006. p. 291.

15. Aronson J. Risk perception in drug therapy. $\mathrm{Br} \mathrm{J}$ Clin Pharmacol. 2006;62:135-7. http://dx.doi.org/10.1111/j.13652125.2006.02739_1.x

16. Vertinsky I, Wehrung DA. Risk perception and drug safety evaluation: Ottawa: Health and Welfare Canada; 1990. p. 1-4.

17. Durrieu G, Hurault C, Damase-Michel C, Montastruc JL. Perception of risk of adverse drug reactions: A 3-year follow-up of a cohort of medical students. Fundam Clin Pharmacol. 2010;24:423-7. http://dx.doi.org/10.1111/j.14728206.2009.00783.x

18. Durrieu G, Hurault C, Bongard V, Damase-Michel C, Montastruc JL. Perception of risk of adverse drug reactions by medical students: Influence of a 1 year pharmacological course. Br J Clin Pharmacol. 2007;64:233-6. http://dx.doi. org/10.1111/j.1365-2125.2007.02882.x

19. Montastuc JL, Bongard V, Lapeyre-Mestre M. Perception of the risk of gastrointestinal adverse drug reactions with non-steroidal anti-inflammatory drugs (including coxibs): Differences among general practitioners, gastroenterologists and rheumatologists. Eur J Clin Pharmacol. 2003;59:685-8. http://dx.doi.org/10.1007/s00228-003-0648-5

20. Bongard V, Ménard-Taché $S$, Bagheri $H$, Kabiri $K$, Lapeyre-Mestre M, Montastruc JL. Perception of the risk of adverse drug reactions: Differences between health professionals and non health professionals. $\mathrm{Br} \mathrm{J}$ Clin Pharmacol. 2002;54:433-6. http://dx.doi.org/10.1046/j.13652125.2002.01674.x

21. Edwards IR. Prevention and pharmacovigilance: What should we do, what can we do? Drug Saf. 2012;35:87-90. http://dx.doi.org/10.2165/11630510-000000000-00000

22. Maxwell SR. How should teaching of undergraduates in clinical pharmacology and therapeutics be delivered and assessed? Br J Clin Pharmacol. 2012;73:893-9. http:// dx.doi.org/10.1111/j.1365-2125.2012.04232.x 
23. Tribiño G, Maldonado C, Segura O, Díaz J. Costos directos y aspectos clínicos de las reacciones adversas a medicamentos en pacientes hospitalizados en el servicio de medicina interna de una institución de tercer nivel de Bogotá. Biomédica. 2006;26:31-41.

24. Pinzón JF, Maldonado C, Díaz JA, Segura O. Costos directos e impacto sobre la morbimortalidad hospitalaria de eventos adversos prevenibles a medicamentos en una institución de tercer nivel de Bogotá. Biomédica. 2011;31:30715. http://dx.doi.org/10.7705/biomedica.v31i3.320

25. Griffin MR. Epidemiology of nonsteroidal anti-inflammatory drug-associated gastrointestinal injury. Am J Med. 1998; 104:23S-9.

26. Durrieu G, Hurault C, Damase-Michel C, Montastruc JL. Perception of risk of adverse drug reactions: A 3-year follow-up of a cohort of medical students. Fundam Clin Pharmacol. 2009:24:423-7. http://dx.doi.org/10.1111/j.14728206.2009.00783.x
27. Ganther JM, Kreling DH. Consumer perceptions of risk and required cost savings for generic prescription drugs. $J$ Am Pharm Assoc (Wash). 2000;40:378-83.

28. Mebane FE. The importance of news media in pharmaceutical risk communication: Proceedings of a workshop. Pharmacoepidemiol Drug Saf. 2005;14:297-306. http://dx. doi.org/10.1002/pds.993

29. Chinnock P, Siegfried N, Clarke M. Is evidence-based medicine relevant to the developing world?: Systematic reviews have yet to achieve their potential as a resource for practitioners in developing countries. Evid Based Complement Alternat Med. 2005;2:321-4. http://dx.doi.org/10. 1093/ecam/neh114

30. Sabri AA, Qayyum MA. The problem of evidence-based medicine in developing countries. CMAJ. 2006;175:62. http://dx.doi.org/10.1503/cmaj.1060108 